\title{
TROP2 increases growth and metastasis of human oral squamous cell carcinoma through activation of the PI3K/Akt signaling pathway
}

\author{
GENXIONG TANG ${ }^{1,2}$, QI TANG $^{3}$, LIZHOU JIA $^{3}$, YUAN CHEN $^{4}$, LIANGYUAN LIN $^{1}$, \\ XINGWANG KUAI ${ }^{5}$, AIXIU GONG ${ }^{1}$ and ZHENGQING FENG ${ }^{2,3,6,7}$
}

\begin{abstract}
${ }^{1}$ Department of Stomatology, Children's Hospital of Nanjing Medical University, Nanjing, Jiangsu 210008; ${ }^{2}$ Department of Pathology, ${ }^{3}$ National Health Commission Key Laboratory of Antibody Technique, Nanjing Medical University, Nanjing, Jiangsu 211166; ${ }^{4}$ Department of Otolaryngology, Second Affiliated Hospital of Nanjing Medical University, Nanjing, Jiangsu 210011; ${ }^{5}$ Department of Basic Medicine, Jiangsu College of Nursing, Huai'an, Jiangsu 223001; ${ }^{6}$ Jiangsu Collaborative Innovation Center for Cancer Personalized Medicine; ${ }^{7}$ Key Laboratory of Cancer Biomarkers, Prevent and Treatment, Cancer Center, Nanjing Medical University, Nanjing, Jiangsu 211166, P.R. China
\end{abstract}

Received February 16, 2019; Accepted September 20, 2019

DOI: $10.3892 /$ ijmm.2019.4378

\begin{abstract}
Most malignant neoplasms of the oral cavity are oral squamous cell carcinoma (OSCC), which is a type of highly malignant tumor with a propensity for forming distant metastases. Trophoblast cell surface antigen 2 (TROP2) is a transmembrane protein that is overexpressed in several types of tumor cells, although its role and regulatory mechanism in OSCC have not been determined. The aim of the present study was to examine the effects of TROP2 in human OSCC cell lines. The present study demonstrated that TROP 2 protein expression was upregulated in OSCC cell lines. Transfection of short hairpin RNA (shRNA) targeting TROP2 (sh-TROP2) reduced cell proliferation, migration and invasion of OSCC cell lines, whereas overexpression of TROP2 increased proliferation, migration and invasion. sh-TROP2 transfection in OSCC cell lines inhibited tumor growth in OSCC mouse models. Furthermore, TROP2 expression activated the phosphoinositide 3-kinase (PI3K)/Akt signaling pathway in human OSCC cells. These results suggest that TROP2 induces cell
\end{abstract}

Correspondence to: Professor Zhengqing Feng, National Health Commission Key Laboratory of Antibody Technique, Nanjing Medical University, 101 Longmian Road, Nanjing, Jiangsu 211166, P.R. China

E-mail: fengzhenqing@njmu.edu.cn

Dr Aixiu Gong, Department of Stomatology, Children's Hospital of Nanjing Medical University, 72 Guangzhou Road, Nanjing, Jiangsu 210008, P.R. China

E-mail: gongax2013@163.com

Key words: trophoblast cell surface antigen 2, oncogene, oral squamous cell carcinoma, phosphoinositide 3-kinase/Akt signaling pathway growth, migration and invasion through activation of the $\mathrm{PI} 3 \mathrm{~K} /$ Akt signaling pathway in OSCC cells.

\section{Introduction}

Oral squamous cell carcinoma (OSCC), a subtype of head and neck cancer, is one of the most common types of tumors and ranks as the eighth leading cause of cancer-associated mortality worldwide (1). Smoking and alcohol consumption are the primary risk factors of OSCC (2). Other possible etiological factors include insufficient oral hygiene, infection with human papillomavirus strains 16 and 18, and chronic oral diseases such as oral leukoplakia or oral erythroplakia (3). Despite significant improvements in diagnostic and therapeutic techniques, OSCC is also prone to early metastasis (4). Patients with OSCC frequently exhibit insensitivity to radiation or chemotherapy, and consequently, patients suffer from ineffective treatment (5). The overall 5-year survival rate of OSCC has remained $<60 \%(6,7)$. OSCC is a multistage process involving the accumulation of dysregulation of numerous oncogenes and tumor suppressor genes, resulting in activation or inhibition of various signaling pathways (8). Therefore, there is a need to identify new oncogenes or tumor suppresser genes, which may be useful as diagnostic and prognostic biomarkers for OSCC, and may provide an understanding of the molecular mechanisms underlying OSCC metastasis. Effective intervention measures to control metastasis have been a primary focus of OSCC research $(9,10)$.

The human trophoblast cell surface antigen 2 (TACSTD2/TROP2/M1S1/GA733-1; henceforth referred to as TROP2) gene is a $35 \mathrm{kDa}$ type I transmembrane glycoprotein with little or no expression in normal tissues and upregulated expression in cancer tissues $(11,12)$. TROP2 is a candidate tumor prognostic marker for predicting poor clinical outcomes, and upregulated expression is associated with tumor recurrence, invasiveness and metastasis (13). Other studies have demonstrated that TROP2 expression is upregulated in 
pancreatic cancer cells and strongly induces mitogen-activated protein kinase activity and metastasis (14-16). TROP2 expression is upregulated in prostate stem cells, which are susceptible to transformation (17). In addition, TROP2 reportedly serves an important role in tumorigenesis of laryngeal carcinoma and may thus be a potential target for treatment; however, its function remains unknown (18). Previously, reverse transcription-quantitative (RT-q)PCR and immunohistochemistry (IHC) were used to study the mRNA and protein expression levels of TROP2, respectively, and it was demonstrated that TROP2 expression was upregulated in OSCC tissues compared with the adjacent normal tissues (19). However, the molecular mechanisms and potential function of TROP2 in OSCC cells remain unclear.

The present study evaluated the expression of TROP 2 in OSCC cell lines, as well as the effects of TROP2 on proliferation, migration, and invasion of the OSCC cells in vitro and in vivo. The effects of TROP2 overexpression or knockdown were examined. The effects of TROP2 on modulation of the phosphoinositide 3-kinase (PI3K) signaling pathway in human OSCC cell lines was also examined using western blot analyses. The results of this study suggest that TROP2 may serve as a potential therapeutic target for the clinical management of OSCC.

\section{Materials and methods}

Cell culture and transfection. The OSCC cell lines, SCC4, HSC3, CAL27 and HN6 were purchased from American Type Culture Collection, and normal oral epithelial cells (HOK) were obtained from the Jiangsu Key Laboratory of Oral Disease (Jiangsu, China). Cells were maintained in RPMI-1640 medium (Invitrogen; Thermo Fisher Scientific, Inc.) supplemented with 10\% FBS (Invitrogen; Thermo Fisher Scientific, Inc.), penicillin $(100 \mathrm{IU} / \mathrm{ml})$ and streptomycin $(100 \mu \mathrm{g} / \mathrm{ml})$ at $37^{\circ} \mathrm{C}$ in a humidified, $5 \% \mathrm{CO}_{2}$ atmosphere. Short hairpin (sh)RNA-TROP2, shRNA-negative control (NC), a TROP2 overexpression vector (OE-TROP2) and a TROP2 empty lentiviral vector control (EV-TROP2) were all purchased from GeneCopoeia, Inc., and cells were transfected for $48 \mathrm{~h}$ using Lipofectamine 2000 transfection reagent (Invitrogen; Thermo Fisher Scientific, Inc.) according to the manufacturer's protocol. The shRNA-TROP2 target sequence was as follows: GTGTCCCACCAACAAGATGAC.

Animal experiments. A total of sixteen BALB/c nude mice (weighing 20-25 g and aged 4 weeks old) were purchased from Beijing Vital River Laboratory Animal Technology Co., Ltd. The animal experiments were performed in accordance with the national guidelines for the care and use of laboratory animals (20). The animal experiments were approved by the Animal Ethics and Welfare Committee of Nanjing Medical University (Nanjing, China; approval no. 1804005). All animals were housed under standard conditions with ad libitum access to food and water at $22-24^{\circ} \mathrm{C}$ and a humidity of $55-70 \%$ on a $12 \mathrm{~h}$ light/dark cycle. Animal rooms were maintained in specific-pathogen-free condition. All animal health and behavior were monitored every 10 days. When the animals exhibited loss of appetite, weakness (inability to eat or drink), clinical symptoms of severe loss of organ function, ineffective treatment or solid tumors $>10 \%$ of the animal's weight, the animals were euthanized. Mice were euthanized using $\mathrm{CO}_{2}$, with an air displacement rate of $20 \%$ of the volume of the container/min. The animals were euthanized in their home cage to avoid stressing the animals. After confirmation of death, cervical dislocation was performed on the mice to ensure death. The euthanasia container was not crowded to allow normal postural adjustments. During euthanasia, all animals were clearly seen through the euthanasia container. The rate of carbon dioxide flow was $2.51 / \mathrm{min}$ avoiding animal distress caused by excessive flow. The authors then observed respiration, corneal reflex and eye color to confirm euthanasia. The criteria for verifying animal death was no breathing, no heartbeat and no corneal reflex. In the experiment, shRNA-TROP2-transfected HN6 cells and lentiviral vector control cells $\left(1 \times 10^{6} / 100 \mu \mathrm{l}\right)$ were injected subcutaneously into the flank of each 4-week-old immunodeficient nude mouse (left flank: TROP2, right flank: Control). Tumor growth was evaluated weekly for 5 weeks. Tumor size was determined by caliper measurement in two perpendicular diameters of the implant every 3 days. The nude mice were subsequently sacrificed, and xenograft tumors were measured. Tumor volumes were calculated using the following formula: $\mathrm{V}=\pi \mathrm{AB}^{2} / 6$, where $\mathrm{A}$ represents the maximum diameter and $\mathrm{B}$ is the perpendicular diameter. Finally, immunostaining of TROP2 protein expression was performed using these tumor tissues.

$R T$ - $q P C R$. RNA was extracted using TRIzol ${ }^{\circledR}$ reagent (Thermo Fisher Scientific, Inc.) and reverse transcribed into cDNA using the PrimeScript ${ }^{\mathrm{TM}}$ RT Reagent kit (Takara Bio, Inc.) according to the manufacturer's protocol. The reverse transcription temperature protocol used was $42^{\circ} \mathrm{C}$ for $50 \mathrm{~min}$ and $95^{\circ} \mathrm{C}$ for $5 \mathrm{~min}$. The primers for TROP2 (All-in-One qPCR Primer) were purchased from GeneCopoeia, Inc., and GAPDH primers (internal control) were purchased from Genscript. The primer sequences were as follows: TROP2 forward, 5'-TGTCCTGATGTGATATGTCTGAG-3' and reverse, 5'-GGGTGAGAGTGGGTTGGG-3'; and GAPDH forward, 5'-GGAGCGAGATCCCTCCAAAAT-3' and reverse, 5'-GGC TGTTGTCATACTTCTCATGG-3'. qPCR was performed on an ABI PRISM 7500HT Sequence Detection system (Applied Biosystems; Thermo Fisher Scientific, Inc.) in 96-well plates. The qPCR thermocycling conditions were: Pre-denaturation at $95^{\circ} \mathrm{C}$ for $10 \mathrm{~min}$, followed by 30 cycles of $95^{\circ} \mathrm{C}$ for $10 \mathrm{~min}$, $56^{\circ} \mathrm{C}$ for $30 \mathrm{sec}$ and $72^{\circ} \mathrm{C}$ for $30 \mathrm{sec}$; with a final extension step at $72^{\circ} \mathrm{C}$ for $30 \mathrm{sec}$. The products were stored at $4^{\circ} \mathrm{C}$ until further use. Relative expression levels were calculated as ratios normalized to GAPDH. The Cq-value for each sample was calculated using the $\Delta \Delta \mathrm{Cq}$ method, and the results were expressed as $2^{-\Delta \Delta \mathrm{Cq}}(21)$. Experiments were performed in triplicate.

Cell Counting Kit-8 (CCK-8) assay. Cells were seeded in a 96-well plate at a density of $5 \times 10^{3}$ cells per well and cultured for 24,48 or $72 \mathrm{~h}$. Cell proliferation assays were performed using a CCK-8 assay (Beyotime Institute of Biotechnology) according to the manufacturer's protocol. Absorbance was subsequently recorded at $450 \mathrm{~nm}$ using an Elx800 Reader (BioTek Instruments, Inc.). 
Flow cytometry analysis of cell cycle distribution and apoptosis. After counting, $1 \times 10^{6} / \mathrm{ml}$ cells were washed with PBS twice and incubated with $5 \mu$ l Annexin V-allophycocyanin-7 aminoactinomyosin $\mathrm{D}$ and $10 \mu \mathrm{l}$ propidium iodide (PI) for $5 \mathrm{~min}$ at room temperature (RT), and then the mixture was incubated at RT for $30 \mathrm{~min}$ in the dark. The trypsinized cells were fixed in $70 \%$ ethanol at $-20^{\circ} \mathrm{C}$ for $24 \mathrm{~h}$, and subsequently washed and incubated with RNaseA (Thermo Fisher Scientific, Inc.) and $1 \mathrm{ml} \mathrm{PI}$ at $4^{\circ} \mathrm{C}$ for $30 \mathrm{~min}$ in the dark. Cell cycle distribution was assessed using ModFit LT version 3.2 (Verity Software House, Inc.), and apoptosis analysis was assessed using CellQuest version 2.0 (BD Biosciences).

Wound-healing assay. Cells were seeded on 6-well plates in culture media at a density of $5 \times 10^{5}$ cells per well. The cells were plated in serum-free media $12 \mathrm{~h}$ prior to assay initiation. The confluent monolayer was scratched using a cell scraper. Serum-free media was added, and cells were incubated for an additional $48 \mathrm{~h}$ at $37^{\circ} \mathrm{C}$ with $5 \% \mathrm{CO}_{2}$ in a humidified chamber. The wound-healing percentage was calculated as the ratio of the wound width at $48 \mathrm{~h}$ to the wound width at $0 \mathrm{~h}$.

Migration and invasion assays. For the Transwell migration assay, $5 \times 10^{4} \mathrm{HN} 6$ or SCC4 cells/well were plated in the top chamber with an uncoated membrane (pore size, $8 \mu \mathrm{m}$; 24-well plate; BD Biosciences). For the invasion assay, Matrigel (BD Biosciences) was polymerized in Transwell inserts for $2 \mathrm{~h}$ at $37^{\circ} \mathrm{C}$ prior to plating the cells. In both assays, cells were plated in the top chamber in serum-free media, and the lower chamber was filled with media supplemented with $10 \%$ FBS. Cells were incubated for $12 \mathrm{~h}$ for the migration assay and $24 \mathrm{~h}$ for the invasion assay at $37^{\circ} \mathrm{C}$ with $5 \% \mathrm{CO}_{2}$ in a humidified chamber. Cells in the upper chamber, which did not migrate or invade through the pores, were carefully removed with a cotton swab. Cells on the lower surface of the membrane were stained with $0.1 \%$ crystal violet for $30 \mathrm{~min}$ at room temperature and counted.

Western blot analysis. Western blotting was performed as previously described (22), and the following antibodies were used: Anti-TROP2 (1:2,000; cat. no. ab205718; Abcam), anti-phosphatase and tensin homolog deleted on chromosome ten (PTEN; 1:1,000; cat. no. ab170941; Abcam), anti-Akt (1:10,000; cat. no. ab179463; Abcam), anti-p-Akt (1:500; cat. no. ab8805; Abcam), anti-PI3K-P85 (1:1,000; cat. no. ab86714; Abcam), anti-pyruvate dehydrogenase kinase 1 (PDK; 1:1,000; cat. no. ab110025; Abcam) and anti-GAPDH (1:10,000; cat. no. ab181602; Abcam). Primary antibodies were diluted in $5 \%$ non-fat milk and the membranes were incubated with the antibodies for $2 \mathrm{~h}$ at room temperature. After washing with PBST three times, the membrane was incubated with secondary antibodies at room temperature for $1 \mathrm{~h}$. Signals were visualized using an enhanced chemiluminescence system (GE Healthcare) and densitometry analysis was performed using ImagePro plus version 6.0 (Media Cybernetics, Inc.). Each experiment was repeated three times.

Immunohistochemical staining. The standard EnVision staining technique was used to examine protein expression (23). Specimens (4- $\mu \mathrm{m}$ tissue sections) were dewaxed and rehydrated in a graded series of alcohol solutions (95, 85 and $75 \%$; 5 min per solution). Endogenous peroxidase was inhibited using 3\% hydrogen peroxide. Antigen retrieval was performed using sodium citrate acid buffer $(\mathrm{pH}$ 6.0) in a microwave oven at $95^{\circ} \mathrm{C}$ for $10 \mathrm{~min}$ and incubated overnight at $4^{\circ} \mathrm{C}$ with TROP2 (1:200; R\&D Systems, Inc.) antibody. The EnVision peroxidase kit (Dako; Agilent Technologies, Inc.) was used to detect the reactions. Every tissue section was incubated at $37^{\circ} \mathrm{C}$ for 5-10 min using 3,3'-diaminobenzidine tetrahydrochloride with hematoxylin as the counterstain, followed by gradient alcohol dehydration (70, 80 and 90\%) and sealed with neutral balsam. As a negative control, the sections were treated with PBS instead of TROP2 antibody. Slides stained with TROP2 antibody were first scanned under a low power magnification (x100) to identify the five areas with the highest TROP2 density (hot spots). These five areas were imaged at a magnification of $x 400$. Staining intensity was scored as follows: 0 , no staining; 1 , weak staining; 2 , moderate staining; or 3, strong staining. The total score was obtained by multiplying the percentage of positive cells in each field $(0-100 \%)$ by the staining intensity score. The sections were assessed separately by two experienced pathologists in a double-blind manner.

Statistical analysis. SPSS version 20.0 (IBM Corp.) was used for statistical analysis. Comparisons between groups were calculated using a one-way ANOVA with a post-hoc SNK test. $\mathrm{P}<0.05$ was considered to indicate a statistically significant difference.

\section{Results}

Expression of TROP2 in human OSCC cell lines. Flow cytometry, RT-qPCR and western blotting were used to investigate TROP2 mRNA and protein expression levels in various types of human OSCC cell lines compared with normal oral epithelial cells. As shown in Fig. 1A, the TROP2 protein expression ratio in normal oral $\mathrm{HOK}$ epithelial cells was $1.4 \%$; whereas in SCC4, HSC3, CAL27 and HN6 cells, the ratio was 9.07, $54.16,63.23$ and $82.93 \%$, respectively. Subsequently, RT-qPCR (Fig. 1B) and western blotting (Fig. 1C) were performed to analyze the expression of TROP 2 in the OSCC cell lines. The results were similar to the flow cytometry results. TROP2 expression in HN6 cells was determined to be the highest in the four OSCC cells assessed, and TROP2 expression of SCC4 cells was the lowest in the four OSCC cells assessed. Therefore, these cell lines were used for all subsequent experiments.

TROP2 induces proliferation, migration and invasion of OSCC cells in vitro. To investigate the biological function of TROP2, a control lentiviral vector (EV-TROP2), a lentiviral vector that induced TROP2 expression (OE-TROP2), a negative control shRNA plasmid (shRNA-NC) and an shRNA plasmid targeting TROP2 (shRNA-TROP2) were used. EV-TROP2 and OE-TROP2 were transfected into the TROP2-deficient human OSCC cell line, SCC4. Fluorescence microscopy and RT-qPCR were used to detect the efficiency of OE-TROP2, and the results revealed that TROP 2 mRNA and protein expression levels were increased in the SCC4 cells following transfection. Conversely, TROP2 mRNA and protein expression levels 
A
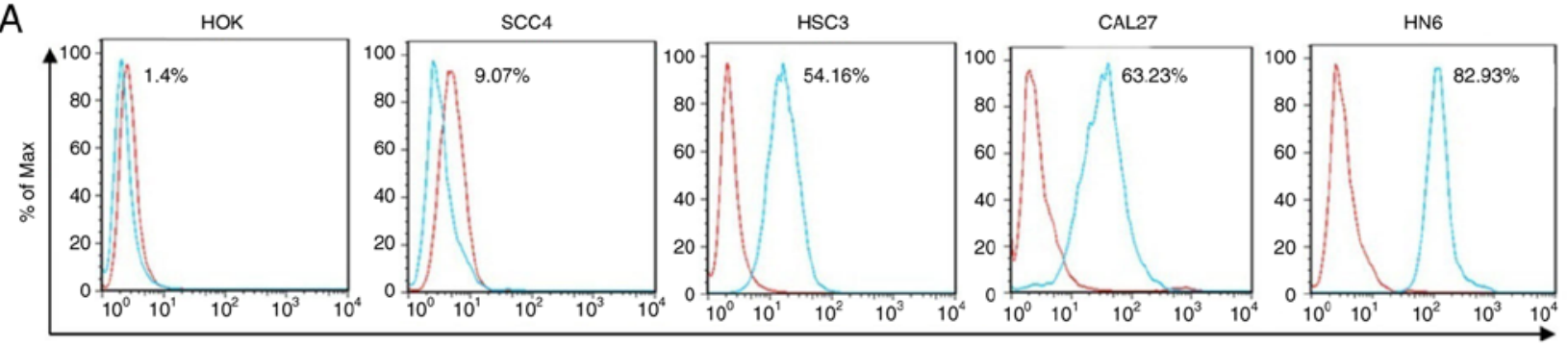

B
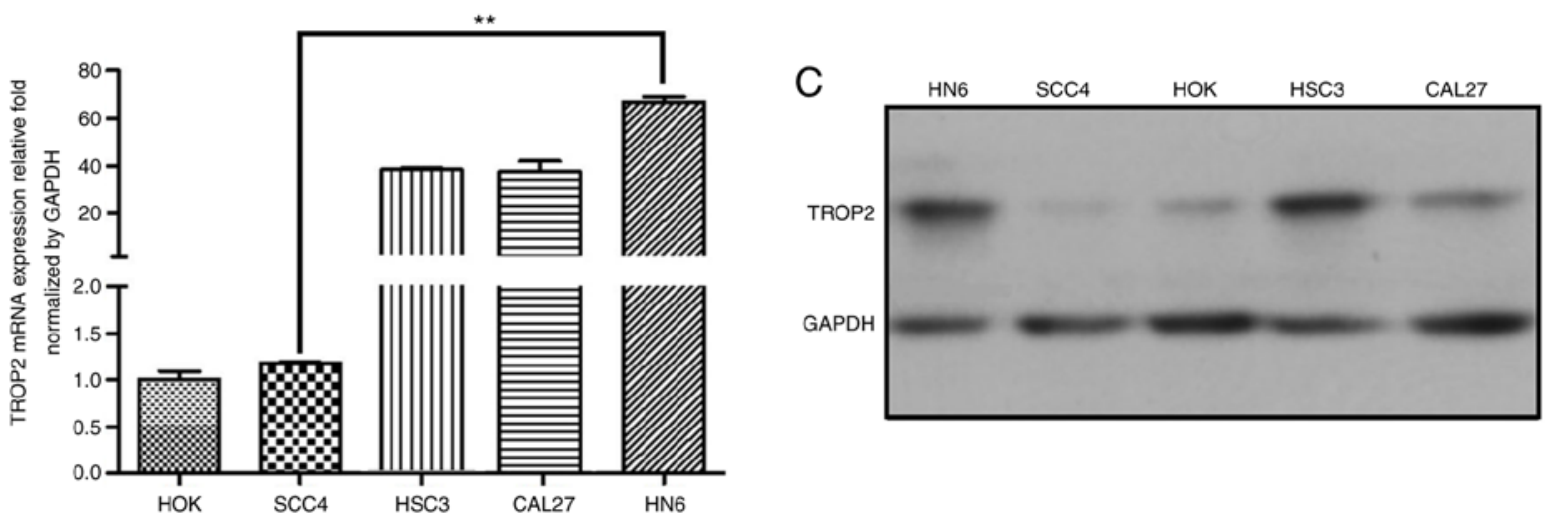

Figure 1. TROP2 protein and mRNA expression levels in various human OSCC cell lines. (A) Protein expression levels of TROP2 were detected by flow cytometry. (B) Expression levels of TROP2 mRNA were detected by reverse transcription-quantitative PCR. ${ }^{* *} \mathrm{P}<0.01$. (C) Expression levels of TROP2 protein were detected by western blotting. TROP2, trophoblast cell surface antigen 2; FL-1, fluorescence-1; FITC, fluorescein isothiocyanate.
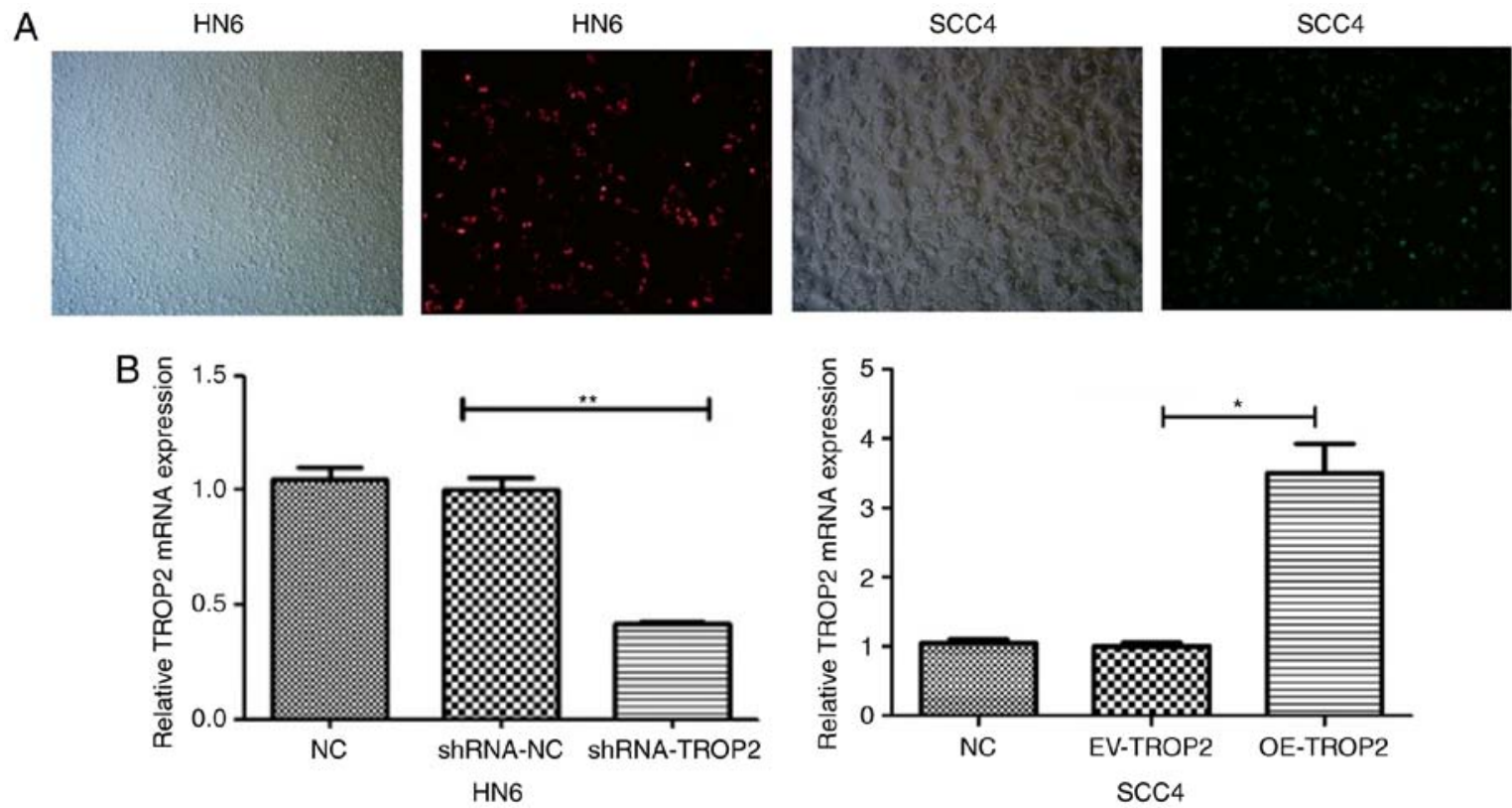

Figure 2. shRNA-TROP2 or OE-TROP2 plasmid transfection efficiency in the OSCC cells. (A) Brightfield and fluorescence images of shRNA-TROP2 or OE-TROP2 plasmid transfection in OSCC cells were analyzed by fluorescence. Left, shRNA-TROP2 plasmid transfected into HN6 cells; Right, OE-TROP2 plasmid transfected into SCC4s. TROP2 mRNA expression levels in (B) HN6 cells transfected with shRNA-TROP2 or SCC4 cells transfected with OE-TROP2. ${ }^{*} \mathrm{P}<0.05,{ }^{* *} \mathrm{P}<0.01$. TROP2, trophoblast cell surface antigen 2 ; sh, short hairpin; OE, overexpression; OSCC, oral squamous cell carcinoma; NC, negative control; EV, empty vector.

were reduced in the cells transfected with shRNA-TROP2 compared with the shRNA-NC group (Fig. 2). A CCK-8 assay was used to examine OSCC cell proliferation at 24,48 and $72 \mathrm{~h}$ following shRNA-TROP2 and OE-TROP2 plasmid transfection. The results showed that TROP2 knockdown significantly reduced cell proliferation in HN6 cells (Fig. 3), whereas TROP2 overexpression increased cell proliferation in SCC4 cells (Fig. 3A). The migration and invasion of OSCC cell lines was investigated following shRNA-TROP2 or OE-TROP2 transfection. Wound-healing assays showed that wound closure was slower in the TROP2 knockdown cells and faster in the cells overexpressing TROP2 suggesting the TROP2 regulated 
A

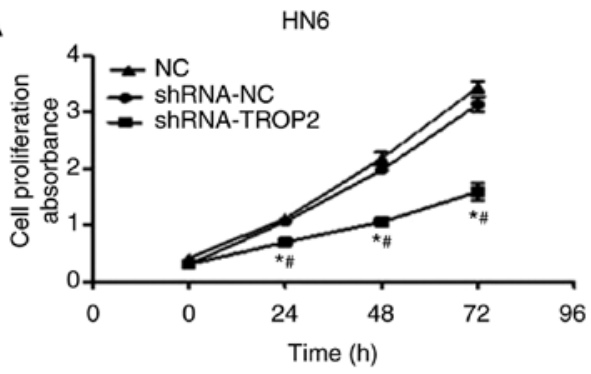

B
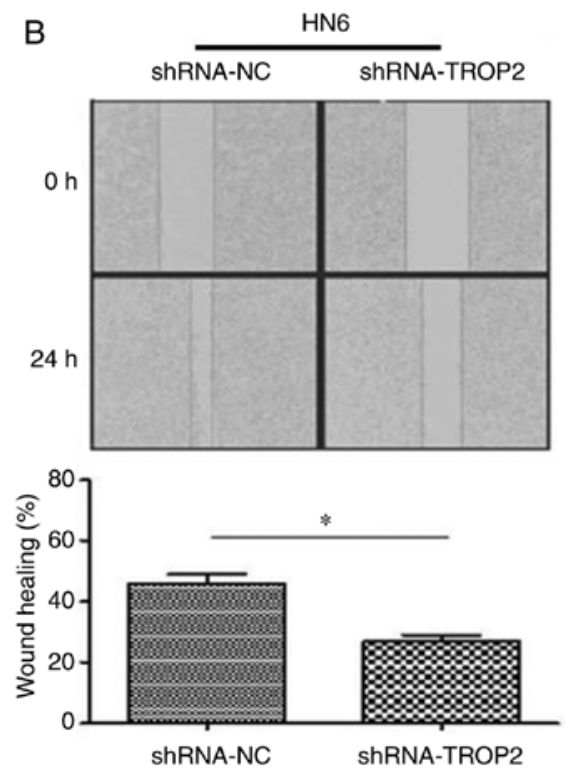

C
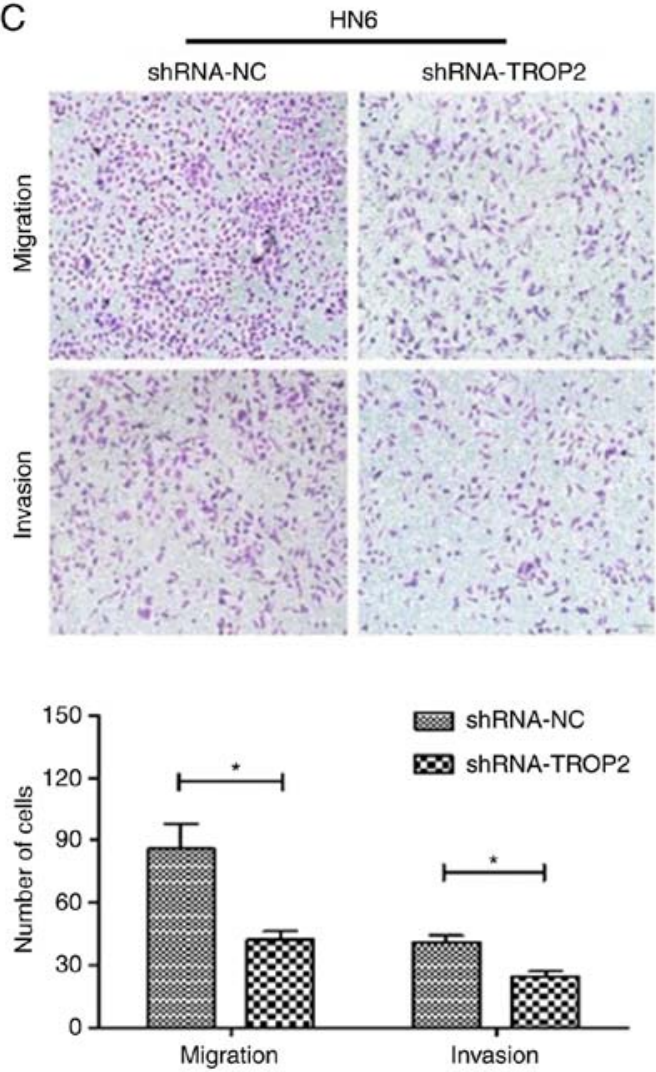
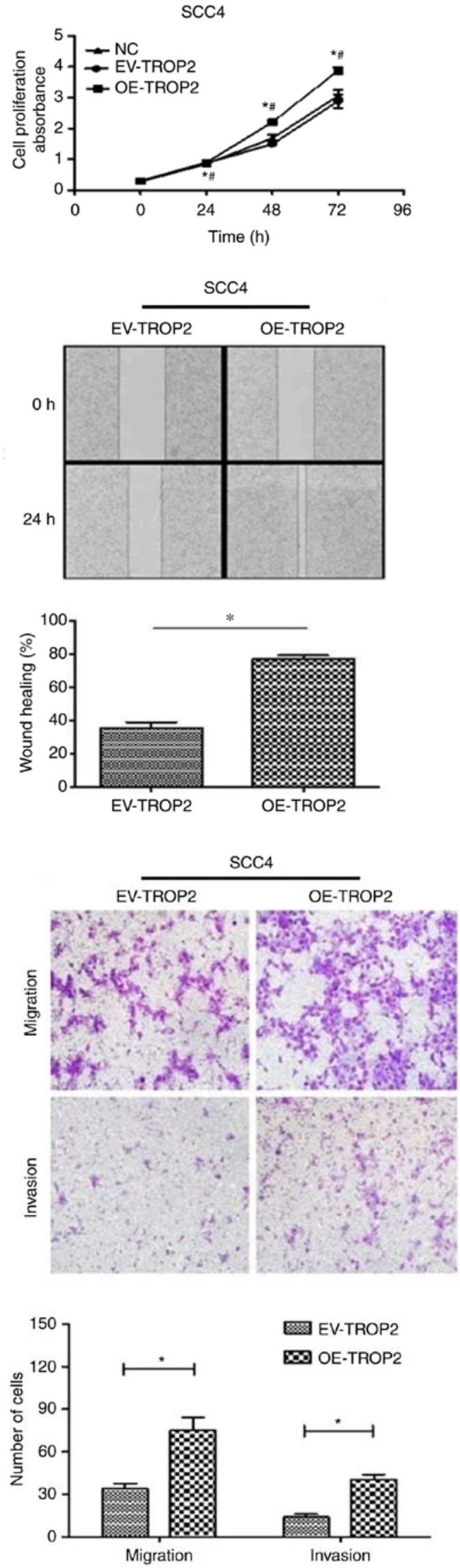

Figure 3. TROP2 increases proliferation, migration and invasion of oral squamous cell carcinoma cells in vitro. (A) Proliferation ratio of HN6 or SCC4 cells transfected with shRNA-TROP2 or OE-TROP2, respectively, after $0,24,48$ and $72 \mathrm{~h} .{ }^{~} \mathrm{P}<0.05$ vs. shRNA-NC or EV-TROP2; " $\mathrm{P}<0.05$ vs. NC group. (B) Migratory capacity of HN6 or SCC4 cells transfected with shRNA-TROP2 or OE-TROP2, respectively. "P<0.05. (C) Migratory and invasive capacity of HN6 or SCC4 cells transfected with shRNA-TROP2 or OE-TROP2, respectively. Data are presented as the mean \pm standard error of the mean. "P<0.05, ${ }^{* *} \mathrm{P}<0.01$. TROP2, trophoblast cell surface antigen 2; sh, short hairpin; OE, overexpression; NC, negative control; EV, empty vector. 
A

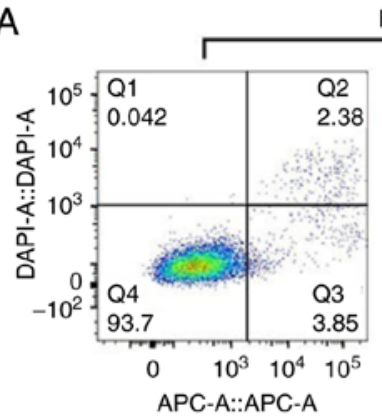

HN6

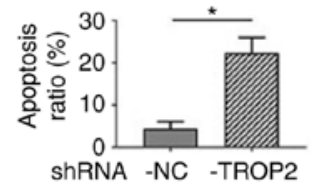

B

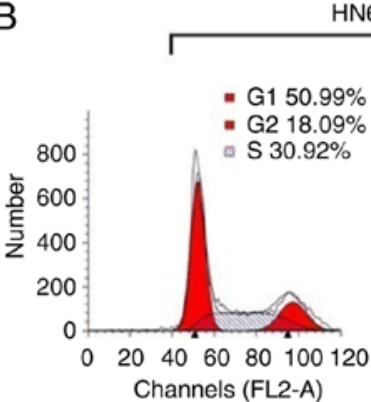

HN6
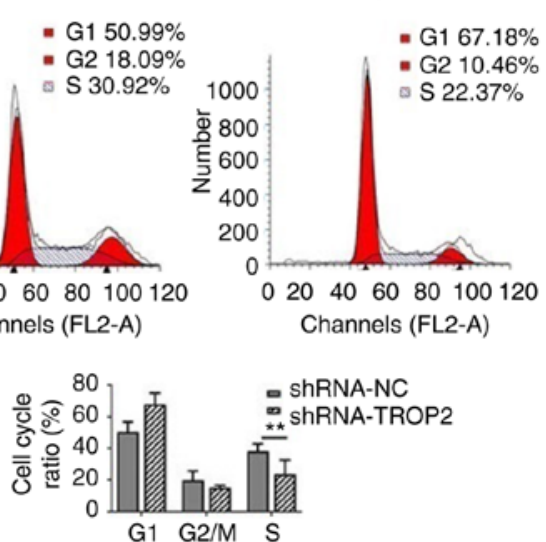
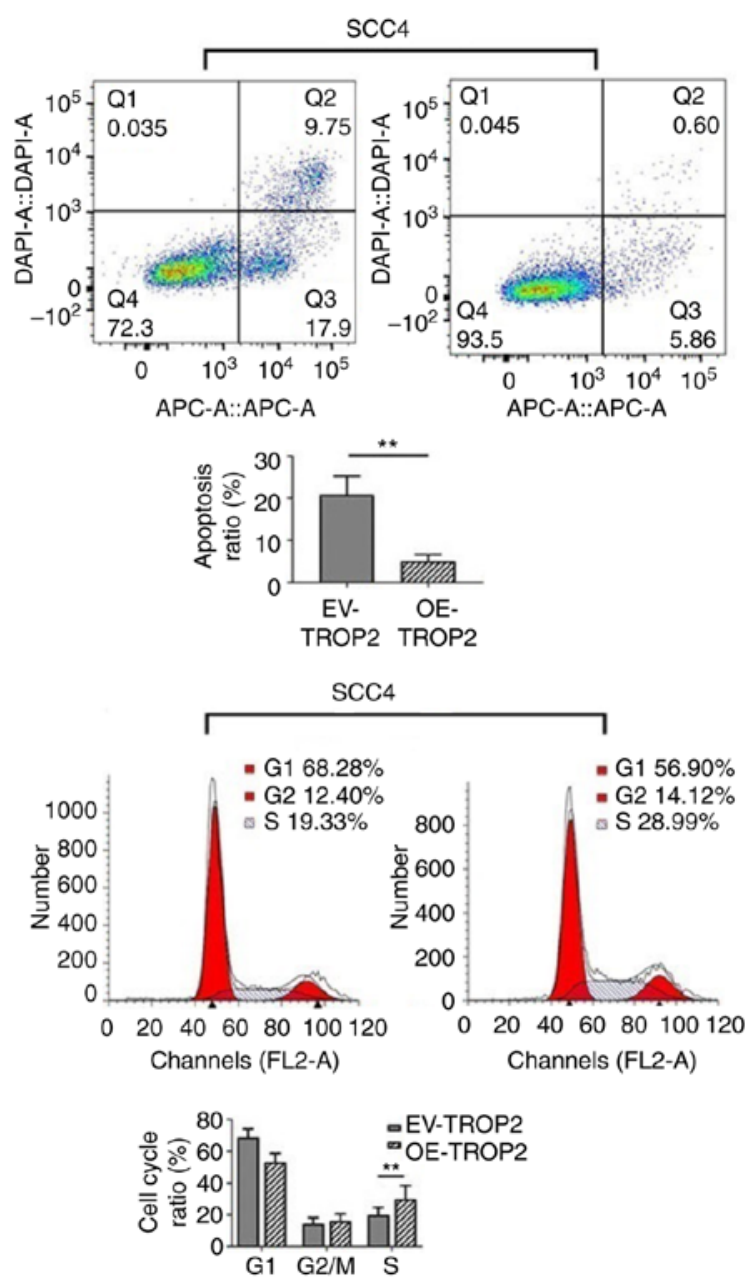

Figure 4. TROP2 induces cell cycle progression and reduces apoptosis in oral squamous cell carcinoma cells in vitro. (A) The proportion of apoptotic HN6 or SCC4 cells transfected with shRNA-TROP2 or OE-TROP2, respectively. (B) Cell cycle phase distribution in HN6 or SCC4 cells transfected with shRNA-TROP2 or OE-TROP2, respectively. Data are presented as the mean \pm standard error of the mean. ${ }^{*} \mathrm{P}<0.05$, ${ }^{* *} \mathrm{P}<0.01$. TROP2, trophoblast cell surface antigen 2; sh, short hairpin; OE, overexpression; $\mathrm{NC}$, negative control; EV, empty vector.

migration (Fig. 3B). For the Transwell assays, the number of cells which had migrated in the shRNA-TROP2-transfected group were counted and the results indicated that after $24 \mathrm{~h}$ of incubation, the number of cells which had migrated was reduced in OSCC cells with knockdown of TROP2. TROP2 knockdown also reduced cell invasion $48 \mathrm{~h}$ after transfection (Fig. 3C); whereas TROP2 overexpression increased migration and invasion of SCC4 cells compared with the negative control-transfected group (Fig. 3C).

TROP2 induces $S$-phase cell cycle progression and inhibits apoptosis in OSCC cells in vitro. Flow cytometry was performed to determine if TROP 2 inhibited apoptosis in OSCC cells. The results showed that the apoptotic ratio was $6.06 \pm 2.06$ in the OE-TROP2 transfected SCC4 cells, which was significantly lower compared with the EV-TROP2 group (26.14 \pm 10.18$)$. Conversely, TROP2 transfection increased apoptosis in HN6 cells significantly compared with the control cells (Fig. 4A). Cell cycle analysis of SCC4 cells transfected with OE-TROP2 or EV-TROP2 was analyzed by flow cytometry. TROP 2 overexpression resulted in a significant increase in the ratio of cells in the $\mathrm{S}$ phase compared with the EV-TROP2 transfected cells. In addition, shRNA-TROP2-treated HN6 cells had significantly fewer cells in the $\mathrm{S}$ phase compared with the control group (Fig. 4B).

Knockdown of TROP2 reduces tumor growth in vivo. To detect the effects of TROP 2 knockdown on cell proliferation in vivo, a nude mouse xenograft model was used. HN6 cells transfected with shRNA-TROP2 or shRNA-NC plasmid were subcutaneously injected into the flanks of nude mice ( $\mathrm{n}=6 ; 1 \times 10^{6}$ cells/mouse). The left sides were subcutaneously injected with HN6 cells transfected with shRNA-TROP2, and the right sides were injected with HN6 cells transfected with shRNA-NC. The mice were sacrificed after 5 weeks, and the average tumor volume and weight were measured (Fig. 5A). The results showed that tumors from the shRNA-TROP2 group grew significantly slower compared with the shRNA-TROP2 tumors (Fig. 5B and C). TROP2 expression was also observed in tumors using IHC staining (Fig. 5D). The results showed that TROP2 protein expression in tumors from the shRNA-TROP2 group was significantly lower compared with the shRNA-NC group. These data suggest that TROP2 can induce tumor growth in vivo.

TROP2 activates the PI3K/Akt signaling pathway in OSCC cell lines. It has been reported TROP 2 may induce cancer cell 
A

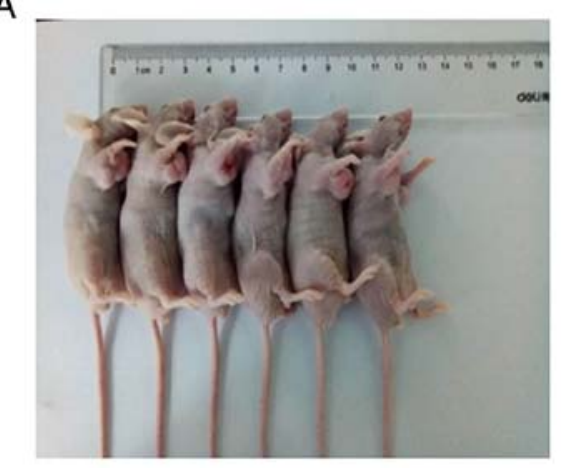

B

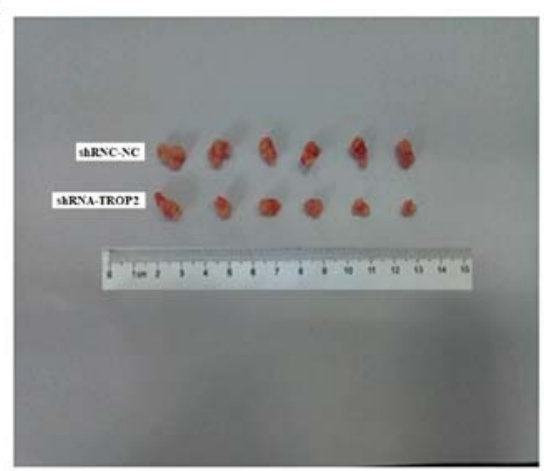

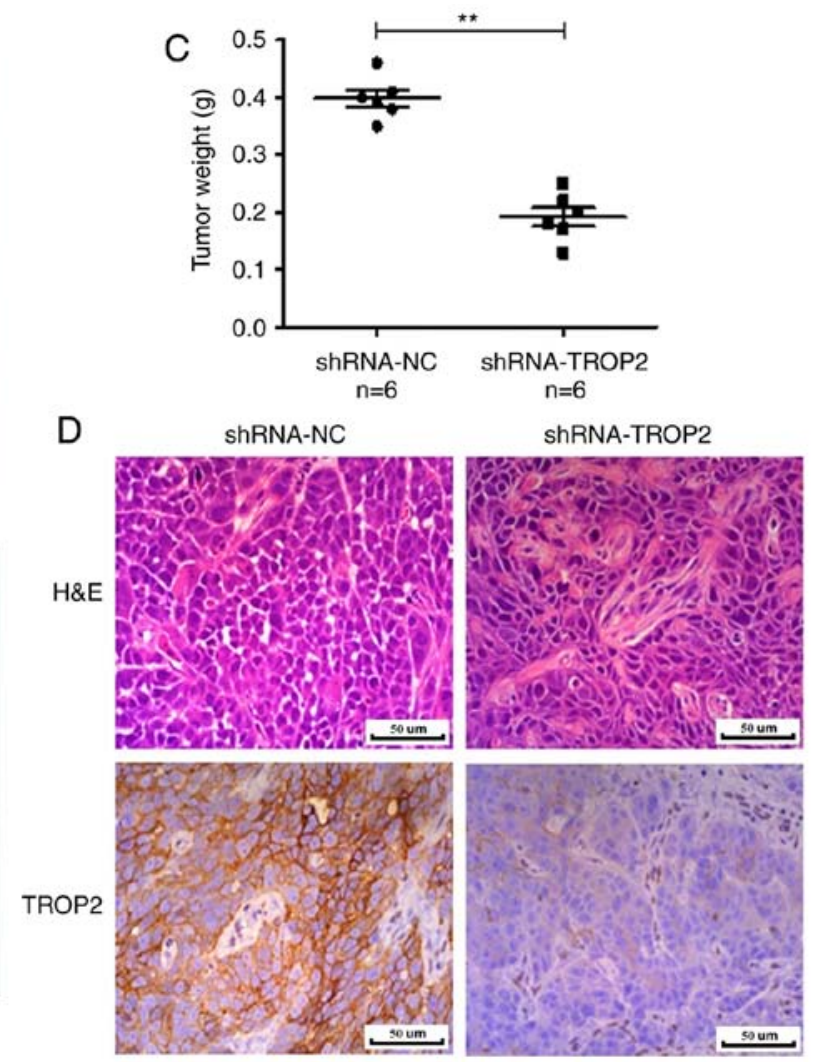

D

Figure 5. Knockdown of TROP2 reduces tumor growth in vivo. (A) Images of tumors in nude mice. (B) Tumor xenografts were removed from the nude mice 5 weeks after inoculation. The top row shows tumors obtained from the control group which were injected with shRNA-NC transfected cells. The bottom row shows tumors obtained from the experimental group which were injected with the shRNA-TROP2 transfected cells. (C) Average tumor weight from nude mice injected with either shRNA-NC or shRNA-TROP2 transfected cells. (D) Representative immunohistochemical images of TROP2 protein expression in tumor tissues from shRNA-NC and shRNA-TROP2 transfected HN6 cells. Data are presented as the mean \pm standard error of the mean. ${ }^{* *} \mathrm{P}<0.01$. TROP2, trophoblast cell surface antigen 2; sh, short hairpin; NC, negative control; H\&E, hematoxylin and eosin.

proliferation through the PI3K/Akt signaling pathway $(24,25)$. Therefore, western blotting was used to monitor the effects of TROP2 on the PI3K/Akt signaling pathway in OSCC cell lines. As shown in Fig. 6, the expression levels of p-Akt, PI3K-P85 and PDK1 were reduced in the shRNA-TROP2 group and PTEN expression was induced compared with the shRNA-NC group. Results from the OE-TROP2 group confirmed that TROP2 activates the PI3K/Akt pathway. In the OE-TROP2 group, p-Akt, PI3K-P85 and PDK1 expression levels were higher and PTEN expression was lower compared with the EV-TROP2 group. No difference was observed in Akt protein expression among the groups. These results suggest that high TROP2 expression in OSCC cells may induce OSCC proliferation, migration and invasion via activation of the PI3K/Akt pathway.

\section{Discussion}

The development and metastasis of OSCC is a complex and multi-step process, spanning progression from dysplasia to carcinoma and involves a variety of epigenetic and genetic changes in the expression of oncogenes and suppressor genes. This process leads to a dysregulation in the balance between cell proliferation, cell death and cell cycle progression (26). Oral tumors with early metastasis to the neck, with local and regional recurrence, and distant metastases are considered to be the leading causes of death in patients with OSCC (27). At present, the treatment options available for patients with OSCC are surgery, adjuvant chemotherapy and radiotherapy; however, the survival rate remains poor (28). Molecular-targeted therapy of tumors is considered a promising treatment strategy, as it may inhibit tumor proliferation and metastasis and promote tumor cell apoptosis by targeting cells with abnormally high expression levels of certain proteins during tumor development (29). For OSCC, early detection, diagnosis, treatment, and identification of tumor markers for the accurate diagnosis and prediction of prognosis are of great significance for the treatment of tumors. Therefore, targeted therapy is a potential treatment direction for improving the survival rates of patients with OSCC.

TROP2 is an oncogene that has been extensively studied in our laboratory $(22,30)$. Studies have shown that TROP2 is upregulated in various types of malignant tumors and is also associated with a poorer prognosis $(14,18,31)$; however, the function of TROP2 in specific pathways remains unclear. The TROP2 cytoplasmic tail is bound to PIP2 and possesses tyrosine/serine phosphorylation sites (32). TROP2 promotes proliferation, migration and metastasis by regulating the PI3K/Akt pathway in gallbladder cancer cells (25). The PI3K/Akt pathway governs cellular growth, proliferation, metabolism and metastasis (33). PTEN negatively regulates the PI3K/Akt signaling way, which is often inactivated by mutations or deletions in different types of cancer $(34,35)$. However, the regulatory mechanisms underlying alterations 

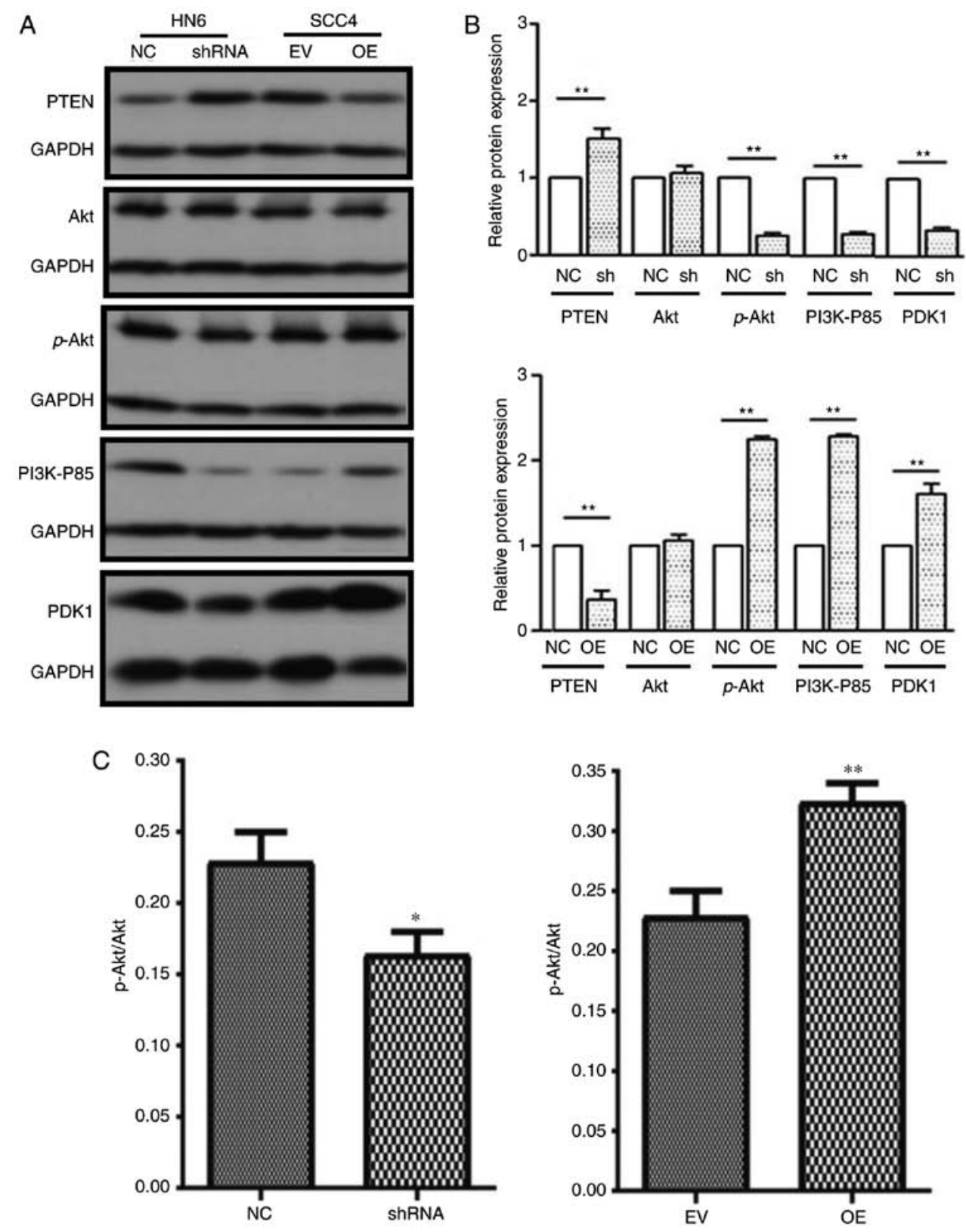

Figure 6. TROP2 activates the PI3K/Akt signaling pathway in oral squamous cell carcinoma cells. (A) Representative western blots of proteins involved in the PI3K/Akt signaling pathway. (B) Densitometry analysis of the protein expression levels of Akt, p-Akt, PI3K-P85, PTEN and PDK1 in shRNA-TROP2 and OE-TROP2 transfected cells, compared with the respective control. (C) Ratio of p-Akt/Akt in the shRNA-TROP2 groups and OE-TROP2 transfected cells, compared with respective control. GAPDH was used as a control. Data are presented as the mean \pm standard error of the mean of three experimental repeats. " $\mathrm{P}<0.05,{ }^{* * *} \mathrm{P}<0.01$. TROP2, trophoblast cell surface antigen 2 ; sh, short hairpin; OE, overexpression; NC, negative control; VE, empty vector; PI3K, phosphoinositide 3-kinase.

in TROP2 expression in OSCC remain to be determined. Therefore, further studies are required to examine the regulatory mechanisms governing TROP2 expression in human OSCC cell lines in vitro and in vivo.

In the present study, cell proliferation was measured and found to be lower in shRNA-TROP2-treated HN6 cell lines compared with the control groups. In 2015, Wang et al (18) found that upregulated expression of TROP2 increased anchorage-independent growth in colon cancer. Furthermore, TROP2 expressed in the membrane of tumor cells has been shown to increase invasion and metastasis of tumor cells (36-38). In the present study, the effects of TROP2 expression were determined by transfecting OE-TROP2 or
shRNA-TROP2 into SCC4 and HN6 cells, and measuring invasion and metastasis. The results indicated that shRNA-TROP2 inhibited the migration and invasion of OSCC cells, whereas OE-TROP2 had the opposite effects. In addition, OE-TROP2 decreased cellular apoptosis and induced S-phase progression in OSCC cell lines, whereas shRNA-TROP2 treatment promoted apoptosis and inhibited S-phase progression. The downregulation of TROP2 was also found to inhibit tumor growth in vivo.

Western blotting was used to identify the regulatory mechanisms of TROP2 in OSCC cell lines. The results showed that TROP2 activated the PI3K/Akt signaling pathway by reducing PTEN expression in OSCC cell lines to facilitate malignant 
progression, consistent with the study by Li et al (25) in gallbladder cancer. PTEN exhibits phosphatase activity and is a known tumor suppressor gene (40). PTEN deregulates the $\mathrm{PI} 3 \mathrm{~K} / \mathrm{PKB} / \mathrm{Akt}$ signaling pathway by dephosphorylating PIP2 and PIP3 in cells (41). The PI3K signaling pathway is an important signaling pathway that reportedly regulates tumor cell proliferation, migration and invasion $(42,43)$. Studies have reported that the PTEN phosphatase is a major negative regulator in this signaling pathway $(44,45)$. The authors also observed that PTEN expression was increased when TROP2 expression was knocked down in shRNA-TROP2 OSCC cell lines and the reverse was true when TROP2 was upregulated in OE-TROP2 OSCC cell lines. Akt is further activated by phosphorylation within the carboxy terminus at Ser473 by PDK1, and PDK1 regulation of the $\mathrm{PI}$ KK/Akt signaling pathway is associated with tumor development (46). PDK1 may thus regulate a series of cell biological functions through the PI3K/Akt signaling pathway, including proliferation, differentiation, apoptosis and metastasis (47). Feng et al (48) showed that the PDK1-Akt signaling pathway activity was directly associated with EMT. In the present study, TROP2 determined to exert its effects on cell biology through the PI3K/Akt signaling pathway was verified.

One limitation of the present study was that the results are based on cell lines and thus should be verified in human samples. Future studies should focus on determination of the functional domain of TROP2 and further explanation of the mechanisms by which TROP2 modulates OSCC cell behavior.

In summary, the results of the present study showed that TROP2 overexpression promotes proliferation, migration and invasion in OSCC cells. In addition, knockdown of TROP2 expression in OSCC cells inhibited tumor growth in OSCC mouse models. Finally, a novel TROP2-PI3K-Akt signaling pathway in OSCC cells was identified. Together, these findings suggest that TROP2 may be an important biomarker in OSCC clinical treatment.

\section{Acknowledgements}

Not applicable.

\section{Funding}

The present study was supported by grants from the Maternal and Child Health Research Projects of Jiangsu Province (grant no. F201607) and the Science and Technology Development Foundation of Nanjing Medical University (Nanjing, China; award no. 2017NJMUZD107).

\section{Availability of data and materials}

The datasets used and or analyzed during the present study are available from the corresponding author upon reasonable request.

\section{Authors' contributions}

ZF and AG conceived and designed the study. GT, LL, YC and XK performed the experiments. LJ analyzed the data and QT contributed to the analysis of data. GT wrote the manuscript. All authors read and approved the final manuscript.

\section{Ethics approval and consent to participate}

The present study obtained ethical approval from the Animal Ethical and Welfare Committee of Nanjing Medical University (Nanjing, China). The animal procedures were performed in accordance with the national guidelines for the care and use of laboratory animals of Nanjing Medical University.

\section{Patient consent for publication}

Not applicable.

\section{Competing interests}

The authors declare that they have no competing interests.

\section{References}

1. Wei J, Wu J, Xu W, Nie H, Zhou R, Wang R, Liu Y, Tang G and Wu J: Salvanic acid B inhibits glycolysis in oral squamous cell carcinoma via targeting PI3K/AKT/HIF-1 $\alpha$ signaling pathway. Cell Death Dis 9: 599, 2018.

2. Montler R, Bell RB, Thalhofer C, Leidner R, Feng Z, Fox BA, Cheng AC, Bui TG, Tucker C, Hoen H and Weinberg A: OX40, PD-1 and CTLA-4 are selectively expressed on tumor-infiltrating $\mathrm{T}$ cells in head and neck cancer. Clin Transl Immunology 5: e70, 2016.

3. Gupta B, Bray F, Kumar N and Johnson NW: Associations between oral hygiene habits, diet, tobacco and alcohol and risk of oral cancer: A case-control study from India. Cancer Epidemiol 51: 7-14, 2017.

4. Sajan T, Murthy S, Krishnankutty R and Mitra J: A rapid, early detection of oral squamous cell carcinoma: Real time PCR based detection of tetranectin. Mol Biol Res Commun 8: 33-40, 2019.

5. Kim SM, Jeong D, Kim MK, Lee SS and Lee SK: Two different protein expression profiles of oral squamous cell carcinoma analyzed by immunoprecpitation high performance liquid chromatog raphy. World J Surg Oncol 15: 151, 2017.

6. Radhika T, Jeddy N, Nithya $S$ and Muthumeenakshi RM: Salivary biomarkers in oral squamous cell carcinoma-an insight. J Oral Biol Craniofac Res 6 (Suppl 1): S51-S54, 2016.

7. Sasahira T, Kirita T and Kuniyasu H: Update of molecular pathobiology in oral cancer: A review. Int J Clin Oncol 19: 431-436, 2014.

8. Mk H, Prince S, Mohan AM, Krishnan KV and Devi A: Association of Notch4 with metastasis in human oral squamous cell carcinoma. Life Sci 156: 38-46, 2016.

9. Dai BW, Yang ZM, Deng P, Chen YR, He ZJ, Yang X, Zhang S, Wu HJ and Ren ZH: HOXC10 promotes migration and invasion via the WNT-EMT signaling pathway in oral squamous cell carcinoma. J Cancer 10: 4540-4551, 2019.

10. Ma H, Li L, Jia L, Gong A, Wang A, Zhang L, Gu M and Tang G: POM121 is identified as a novel prognostic marker of oral squamous cell carcinoma. J Cancer 10: 4473-4480, 2019.

11. Zhao W, Zhu H, Zhang S, Yong H, Wang W, Zhou Y, Wang B, Wen J, Qiu Z, Ding G, et al: Trop2 is overexpressed in gastric cancer and predicts poor prognosis. Oncotarget 7: 6136-6145, 2016.

12. Lin H, Zhang H, Wang J, Lu M, Zheng F, Wang C, Tang X, $\mathrm{Xu}$ N, Chen R, Zhang D, et al: A novel human Fab antibody for Trop2 inhibits breast cancer growth in vitro and in vivo. Int $\mathrm{J}$ Cancer 134: 1239-1249, 2014

13. Fang YJ, Lu ZH, Wang GQ, Pan ZZ, Zhou ZW, Yun JP, Zhang MF and Wan DS: Elevated expressions of MMP7, TROP2, and survivin are associated with survival, disease recurrence, and liver metastasis of colon cancer. Int J Colorectal Dis 24: 875-884, 2009.

14. Cubas R, Zhang S, Li M, Chen C and Yao Q: Trop2 expression contributes to tumor pathogenesis by activating the ERK MAPK pathway. Mol Cancer 9: 253, 2010.

15. Mao Y, Wang X, Zheng F, Wang C, Tang Q, Tang X, Xu N, Zhang $\mathrm{H}$, Zhang D, Xiong L, et al: The tumor-inhibitory effectiveness of a novel anti-Trop2 Fab conjugate in pancreatic cancer. Oncotarget 7: 24810-24823, 2016. 
16. Fong D, Moser P, Krammel C, Gostner JM, Margreiter R, Mitterer M, Gastl G and Spizzo G: High expression of TROP2 correlates with poor prognosis in pancreatic cancer. Br J Cancer 99: 1290-1295, 2008 .

17. Goldstein AS, Huang J, Guo C, Garraway IP and Witte ON: Identification of a cell of origin for human prostate cancer. Science 329: 568-571, 2010.

18. Wang XD, Wang Q, Chen XL, Huang JF, Yin Y, Da P and Wu H: Trop2 inhibition suppresses the proliferation and invasion of laryngeal carcinoma cells via the extracellular signal-regulated kinase/mitogen-activated protein kinase pathway. Mol Med Rep 12: 865-870, 2015.

19. Tang G, Tang Q, Jia L, Xia S, Li J, Chen Y, Li H, Ding X, Wang F, Hou D, et al: High expression of TROP2 is correlated with poor prognosis of oral squamous cell carcinoma. Pathol Res Pract 214: $1606-1612,2018$

20. Workman P, Aboagye EO, Balkwill F, Balmain A, Bruder G, Chaplin DJ, Double JA, Everitt J, Farningham DA, Glennie MJ, et al: Guidelines for the welfare and use of animals in cancer research. Br J Cancer 102: 1555-1577, 2010.

21. Livak KJ and Schmittgen TD: Analysis of relative gene expression data using real-time quantitative PCR and the 2(-Delta Delta $\mathrm{C}(\mathrm{T})$ ) method. Methods 25: 402-408, 2001.

22. Zhao W, Kuai X, Zhou X, Jia L, Wang J, Yang X, Tian Z, Wang X, Lv Q, Wang B, et al: Trop2 is a potential biomarker for the promotion of EMT in human breast cancer. Oncol Rep 40: 759-766, 2018

23. Hashmi AA, Ali R, Hussain ZF, Faridi N, Khan EY, Bakar SMA Edhi MM and Khan M: Mismatch repair deficiency screening in colorectal carcinoma by a four-antibody immunohistochemical panel in Pakistani population and its correlation with histopathological parameters. World J Surg Oncol 15: 116, 2017.

24. Gu QZ, Nijiati A, Gao X, Tao KL, Li CD, Fan XP and Tian Z: TROP2 promotes cell proliferation and migration in osteosarcoma through PI3K/AKT signaling. Mol Med Rep 18: 1782-1788, 2018.

25. Li X, Teng S, Zhang Y, Zhang W, Zhang X, Xu K, Yao H, Yao J, Wang $\mathrm{H}$, Liang $\mathrm{X}$ and $\mathrm{Hu} \mathrm{Z}$ : TROP2 promotes proliferation, migration and metastasis of gallbladder cancer cells by regulating PI3K/AKT pathway and inducing EMT. Oncotarget 8 : 47052-47063, 2017.

26. Kong XP, Yao J, Luo W, Feng FK, Ma JT, Ren YP, Wang DL and $\mathrm{Bu}$ RF: The expression and functional role of a FOXC1 related mRNA-lncRNA pair in oral squamous cell carcinoma. Mol Cell Biochem 394: 177-186, 2014.

27. Xia X, Du R, Zhao L, Sun W and Wang X: Expression of AEG-1 and microvessel density correlates with metastasis and prognosis of oral squamous cell carcinoma. Hum Pathol 45: 858-865, 2014

28. Chen CJ, Hsu LS, Lin SH, Chen MK, Wang HK, Hsu JD, Lee H and Yeh KT: Loss of nuclear expression of Krüppel-like factor 4 is associated with poor prognosis in patients with oral cancer. Hum Pathol 43: 1119-1125, 2012.

29. Imani R, Seyedmajidi M, Ghasemi N, Moslemi D, Shafaee S and Bijani A: HLA-G expression is associated with an unfavorable prognosis of oral squamous cell carcinoma. Asian Pac J Cancer Prev 19: 2527-2533, 2018

30. Zhao W, Jia L, Kuai X, Tang Q, Huang X, Yang T, Qiu Z, Zhu J, Huang J, Huang $\mathrm{W}$ and Feng Z: The role and molecular mechanism of Trop2 induced epithelial-mesenchymal transition through mediated $\beta$-catenin in gastric cancer. Cancer Med 8 : $1135-1147,2019$.

31. Wanger TM, Dewitt S, Collins A, Maitland NJ, Poghosyan Z and Knauper V: Differential regulation of TROP2 release by PKC isoforms through vesicles and ADAM17. Cell Signal 27: $1325-1335,2015$
32. Cubas R, Li M, Chen C and Yao Q: Trop2: A possible therapeutic target for late stage epithelial carcinomas. Biochim Biophys Acta 1796: 309-314, 2009.

33. Ersahin T, Tuncbag N and Cetin-Atalay R: The PI3K/AKT/mTOR interactive pathway. Mol Biosyst 11: 1946-1954, 2015.

34. Lim HJ, Crowe P and Yang JL: Current clinical regulation of $\mathrm{PI} 3 \mathrm{~K} / \mathrm{PTEN} / \mathrm{Akt} / \mathrm{mTOR}$ signalling in treatment of human cancer. J Cancer Res Clin Oncol 141: 671-689, 2015.

35. Carnero A, Blanco-Aparicio C, Renner O, Link W and Leal JF: The PTEN/PI3K/AKT signalling pathway in cancer, therapeutic implications. Curr Cancer Drug Targets 8: 187-198, 2008.

36. Ju X, Jiao X, Ertel A, Casimiro MC, Di Sante G, Deng S, Li Z, Di Rocco A, Zhan T, Hawkins A, et al: v-Src oncogene induces Trop2 proteolytic activation via cyclin D1. Cancer Res 76: 6723-6734, 2016.

37. Li Z, Jiang X and Zhang W: TROP2 overexpression promotes proliferation and invasion of lung adenocarcinoma cells. Biochem Biophys Res Commun 470: 197-204, 2016.

38. Liu X, Li S and Yi F: Trop2 gene: A novel target for cervical cancer treatment. J Cancer Res Clin Oncol 140: 1331-1341, 2014

39. Salmena L, Carracedo A and Pandolfi PP: Tenets of PTEN tumor suppression. Cell 133: 403-414, 2008.

40. Zhang Y, Zhang R, Luo G and Ai K: Long noncoding RNA SNHG1 promotes cell proliferation through PI3K/AKT signaling pathway in pancreatic ductal adenocarcinoma. J Cancer 9: 2713-2722, 2018.

41. Correction: PI3K inhibition reduces mammary tumor growth and facilitates antitumor immunity and anti-PD1 responses. Clin Cancer Res 24: 3782, 2018

42. Zhang XR, Wang SY, Sun W and Wei C: Isoliquiritigenin inhibits proliferation and metastasis of MKN28 gastric cancer cells by suppressing the PI3K/AKT/mTOR signaling pathway. Mol Med Rep 18: 3429-3436, 2018

43. Wise HM, Hermida MA and Leslie NR: Prostate cancer, PI3K, PTEN and prognosis. Clin Sci (Lond) 131: 197-210, 2017.

44. Yuan B, Zou M,Zhao Y,Zhang K, Sun Y and Peng X: Up-regulation of miR-130b-3p activates the PTEN/PI3K/AKT/NF-кB pathway to defense against mycoplasma gallisepticum (HS Strain) infection of chicken. Int J Mol Sci 19: E2172, 2018

45. Li JW, Wang XY, Zhang X, Gao L, Wang LF and Yin XH: (-)-Epicatechin protects against myocardial ischemiainduced cardiac injury via activation of the PTEN/PI3K/AKT pathway. Mol Med Rep 17: 8300-8308, 2018.

46. Yoshizaki H, Mochizuki N, Gotoh Y and Matsuda M: Akt-PDK1 complex mediates epidermal growth factor-induced membrane protrusion through Ral activation. Mol Biol Cell 18: 119-128, 2007.

47. Vander Broek R, Mohan S, Eytan DF, Chen Z and Van Waes C: The PI3K/Akt /mTOR axis in head and neck cancer: Functions, aberrations, cross-talk, and therapies. Oral Dis 21: 815-825, 2015.

48. Feng Q, Di R, Tao F, Chang Z, Lu S, Fan W, Shan C, Li X and Yang Z: PDK1 regulates vascular remodeling and promotes epithelial-mesenchymal transition in cardiac development. Mol Cell Biol 30: 3711-3721, 2010.

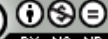

This work is licensed under a Creative Commons Attribution-NonCommercial-NoDerivatives 4.0 International (CC BY-NC-ND 4.0) License. 